\title{
Unsteady Analyses of a Control Valve due to Fluid-Structure Coupling
}

\author{
Yudong Xie, ${ }^{1,2}$ Yong Wang, ${ }^{1,2}$ Yanjun Liu, ${ }^{1,2}$ Fang Cao,, and Yongcheng Pan ${ }^{1,2}$ \\ ${ }^{1}$ School of Mechanical Engineering, Shandong University, Jingshi Road 17923, Jinan, Shandong 250061, China \\ ${ }^{2}$ Key Laboratory of High-Efficiency and Clean Mechanical Manufacture, Shandong University, Ministry of Education, Jinan, \\ Shandong 250061, China
}

Correspondence should be addressed to Yudong Xie; yudong_2177@163.com

Received 11 July 2013; Revised 27 August 2013; Accepted 27 August 2013

Academic Editor: Waqar Khan

Copyright (c) 2013 Yudong Xie et al. This is an open access article distributed under the Creative Commons Attribution License, which permits unrestricted use, distribution, and reproduction in any medium, provided the original work is properly cited.

\begin{abstract}
Control valves play important roles in the control of the mixed-gas pressure in the combined cycle power plants (CCPP). In order to clarify the influence of coupling between the structure and the fluid system at the control valve, the coupling mechanism was presented, and the numerical investigations were carried out. At the same operating condition in which the pressure oscillation amplitude is greater when considering the coupling, the low-order natural frequencies of the plug assembly of the valve decrease obviously when considering the fluid-structure coupling action. The low-order natural frequencies at $25 \%$ valve opening, $50 \%$ valve opening, and $75 \%$ valve opening are reduced by $11.1 \%, 7.0 \%$, and $3.8 \%$, respectively. The results help understand the processes that occur in the valve flow path leading to the pressure control instability observed in the control valve in the CCPP.
\end{abstract}

\section{Introduction}

The steel mills generate vast amounts of blast furnace gas $(\mathrm{BFG})$ and coke-oven gas (COG) in the production. In order to reduce the environmental pollution, some steel mills mix BFG with COG and build combined cycle power plants (CCPP) to make use of the gas [1]. For the normal operation of CСPP, the pressure of mixed gas delivered to the gas turbine should be kept in a steady range.

In CCPP, control valves play important roles in the control of the mixed-gas pressure. The signal of mixed-gas pressure measured using the pressure meter is compared to the signal of the desired pressure by the controller. The controller output accordingly adjusts the opening/closing actuator of the control valve in order to maintain the actual pressure close to the desired pressure. The opening of the control valve depends on the flow forces and the driving forces of the control-valve actuator, while the flow forces and the driving forces are affected by the valve opening. Therefore, there is strong coupling interaction between the fluid and the control valve structure.

According to Morita et al. (2007) and Yonezawa et al. (2008), the typical flow pattern around the control valve is transonic [2,3]. When pressure fluctuations occur, large static and dynamic fluid forces will act on the valves. Consequently, problematic phenomena, such as valve vibrations and loud noises, can occur, with the worst cases resulting in damage of the valve plug and seal [4]. In order to understand the underlying physics of flow-induced vibrations in a steam control valve head, experimental investigations described by Yonezawa et al. (2012) are carried out. Misra et al. (2002) reported that the self-excited vibration of a piping system occurs due to the coincidence of water hammer, acoustic feedback in the downstream water piping, high acoustic resistance at the control valve, and negative hydraulic stiffness at the control valve [5]. Araki et al. (1981) reported that the steam control-valve head oscillation mechanism was forced vibration, while self-excited vibration was not observed [6].

Those studies cited previously are mainly aimed at the modeling of the self-excited vibration, the analysis of vibration parameters stability, and so on [7-11]. Whereas, the studies on the influence of nonlinear fluid-structure coupling of control valve on the valve control characteristics, such as the pressure regulation feature, are still very limited [1217]. In the CCPP, the valve control characteristics affected by the fluid-structure coupling are particularly important for 
the stability of the mixed-gas pressure control. It has not been uncommon to see that the instability of the mixed-gas pressure causes a severe disturbance or even an emergency shutdown of the whole plant, and the handling of such an emergency often becomes a source of new problems and confusion. In this paper, numerical investigations are carried out to clarify the influence of fluid-structure coupling of control valve on not only the flow field but also the gas pressure regulation and the natural frequency changes of the control valve. This study helps understand the processes that occur in the valve flow path leading to the mixed-gas pressure pulsations, which is valuable for the pressure stability control of the mixed gas in the CCPP.

\section{Fluid-Structure Coupling Mechanism of Control Valve}

When the mixed gas passes through the control valve, the gas pressure and flow rate change with the valve opening, as shown in Figure 1. $p_{1}$ and $p_{2}$ express the inlet pressure and outlet pressure of mixed gas, respectively. $\widehat{v}_{1}$ and $\widehat{v}_{2}$ denote the inlet flow rate and outlet flow rate of mixed gas, respectively.

The flow infinitesimal of mixed gas is shown in Figure 2. According to the law of conservation of mass, we can get

$$
\begin{aligned}
& \frac{\partial \rho}{\partial t}+\frac{\rho}{A_{a x}} \frac{\partial A_{a x}}{\partial t}+u \frac{\partial \rho}{\partial x}+\frac{\rho u}{A_{a x}} \frac{\partial A_{a x}}{\partial x}+\rho \frac{\partial u}{\partial x}=0, \\
& \frac{\partial \rho}{\partial t}+\frac{\rho}{A_{a y}} \frac{\partial A_{a y}}{\partial t}+v \frac{\partial \rho}{\partial y}+\frac{\rho v}{A_{a y}} \frac{\partial A_{a y}}{\partial y}+\rho \frac{\partial v}{\partial y}=0, \\
& \frac{\partial \rho}{\partial t}+\frac{\rho}{A_{a z}} \frac{\partial A_{a z}}{\partial t}+w \frac{\partial \rho}{\partial z}+\frac{\rho w}{A_{a z}} \frac{\partial A_{a z}}{\partial z}+\rho \frac{\partial w}{\partial z}=0,
\end{aligned}
$$

where $A_{a x}, A_{a y}$, and $A_{a z}$ represent the $x$-direction crosssectional area, $y$-direction cross-sectional area, and $z$ direction cross-sectional area of the flow infinitesimal, respectively. $\rho$ expresses the mixed-gas density. $u, v$, and $w$ denote $x$-direction velocity component, $y$-direction velocity component, and $z$-direction velocity component, respectively.

According to the balance equation of dynamic flow, we can obtain

$$
\begin{gathered}
\frac{\partial p}{\partial x}+\rho\left[\frac{\partial u}{\partial t}+u \frac{\partial u}{\partial x}+v \frac{\partial u}{\partial y}+w \frac{\partial u}{\partial z}\right]=0, \\
\frac{\partial p}{\partial y}+\rho\left[\frac{\partial v}{\partial t}+u \frac{\partial v}{\partial x}+v \frac{\partial v}{\partial y}+w \frac{\partial v}{\partial z}\right]=0, \\
\frac{\partial p}{\partial z}+\rho g+\rho\left[\frac{\partial w}{\partial t}+u \frac{\partial w}{\partial x}+v \frac{\partial w}{\partial y}+w \frac{\partial w}{\partial z}\right]=0 .
\end{gathered}
$$

According to the equation of flow continuity, we have

$$
\frac{\partial u}{\partial x}+\frac{\partial v}{\partial y}+\frac{\partial w}{\partial z}+\frac{1}{K} \frac{\partial p}{\partial t}=0
$$

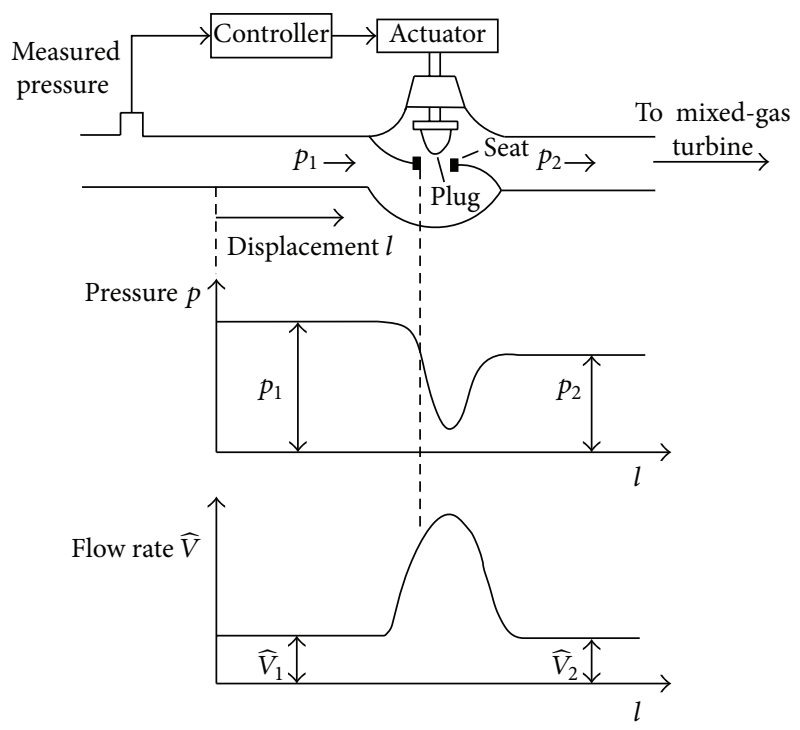

Figure 1: Pressure and flow rate change when the fluid passes through the control valve.

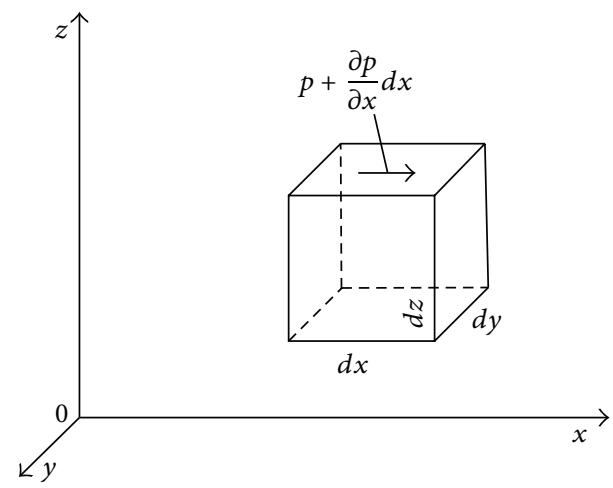

FIgURE 2: Flow infinitesimal.

Based on (1) (3), the flow equation of mixed gas in control valve can be described as

$$
\frac{\partial^{2} p}{\partial x^{2}}+\frac{\partial^{2} p}{\partial y^{2}}+\frac{\partial^{2} p}{\partial z^{2}}-\frac{\rho}{K} \frac{\partial^{2} p}{\partial^{2} t}=0
$$

where $K$ is the bulk modulus of elasticity of the flow.

The discrete pressure distribution of mixed-gas flow field, using Galerkin method, can be expressed as follows:

$$
\Psi(x, y, z, t)=\Upsilon^{T}(x, y, z) \vec{p}(t)=\sum_{m=1}^{M} r_{m}(x, y, z) p_{m}(t),
$$

where $\Upsilon(x, y, z)$ is the shape function matrix and $\vec{p}(t)$ is the pressure vector. $\Upsilon(x, y, z)$ can be written as

$$
\Upsilon(x, y, z)=\left[\begin{array}{c}
r_{1}(x, y, z) \\
r_{2}(x, y, z) \\
\vdots \\
r_{m}(x, y, z)
\end{array}\right] .
$$


$\vec{p}(t)$ can be described as

$$
\vec{p}(t)=\left[\begin{array}{c}
p_{1}(t) \\
p_{2}(t) \\
\vdots \\
p_{m}(t)
\end{array}\right]
$$

Then

$$
\frac{\partial^{2} \Psi}{\partial x^{2}}+\frac{\partial^{2} \Psi}{\partial y^{2}}+\frac{\partial^{2} \Psi}{\partial z^{2}}-\frac{\rho}{K} \ddot{\Psi}=R,
$$

where $R$ is the residual part. The value choice of $\Psi$ should make the value of $R$ get the minimum. Using Galerkin method, $\Psi$ can be calculated as

$$
\begin{gathered}
\iiint_{\Omega} \Upsilon\left(\frac{\partial^{2} \Psi}{\partial x^{2}}+\frac{\partial^{2} \Psi}{\partial y^{2}}+\frac{\partial^{2} \Psi}{\partial z^{2}}\right) d \Omega \\
-\frac{\rho}{K} \iiint_{\Omega} \Upsilon \Psi d \Omega=0 .
\end{gathered}
$$

The discrete flow equation of mixed gas can be described as

$$
\begin{aligned}
& \left(\frac{\rho}{K} \iiint_{\Omega} \Upsilon \Upsilon^{T} d \Omega\right) \frac{\partial^{2} \vec{p}}{\partial t^{2}}+\left(\frac{1}{\sqrt{K / \rho}} \iint_{S_{r}} \Upsilon \Upsilon^{T} d S_{r}\right) \frac{\partial \vec{p}}{\partial t} \\
& \quad+\left(\iiint_{\Omega} \nabla \Upsilon \cdot \nabla \Upsilon^{T} d \Omega\right) \vec{p}+\rho\left(\iint_{S_{i}} \Upsilon_{s}^{T} d S_{i}\right) \Lambda \frac{\partial^{2} \Gamma}{\partial t^{2}} \\
& \quad=F_{i},
\end{aligned}
$$

where $\Gamma$ is the displacement vector, $\Lambda$ represents the coordinate transformation matrix, $F_{i}$ denotes the input exciting vector, $\Omega$ expresses the flow domain volume, $\Upsilon_{s}$ is the insertion function vector of structure system, $S_{i}$ is the surface area of the fluid-structure edge, and $S_{r}$ denotes the surface area of the boundary of flange interface of control valve.

The motion equation of structure domain can be written as

$$
M \frac{\partial^{2} \Gamma}{\partial t^{2}}+C \frac{\partial \Gamma}{\partial t}+K_{F} \Gamma+F_{e}=F_{s},
$$

where $M$ is the mass matrix of structure, $C$ denotes the damping matrix, $K_{F}$ is the structure stiffness matrix, $F_{e}$ expresses the flow-nodal force vector of the fluid-structure edge, and $F_{s}$ is the external exciting vector.

In the fluid-structure edge, the generalized normal force vector of the flow infinitesimal is as follows:

$$
F_{e}^{*}=-\Lambda^{T}\left(\iint_{S_{\mathrm{ie}}} \Upsilon_{\mathrm{en}} \Upsilon_{\mathrm{sen}}^{T} d S_{\mathrm{ie}}\right) \vec{p}_{\mathrm{en}},
$$

where $\Upsilon_{\mathrm{en}}$ is the shape function vector of the structure infinitesimal, $S_{\text {ie }}$ is the surface area of the fluid-structure edge of the infinitesimal, $\Upsilon_{\text {sen }}$ denotes the shape function vector of the flow infinitesimal, and $\vec{p}_{\text {en }}$ is the pressure vector of the flow infinitesimal.
From (11) and (12), we can get

$$
\begin{aligned}
M \frac{\partial^{2} \Gamma}{\partial t^{2}} & +C \frac{\partial \Gamma}{\partial t}+K_{F} \Gamma \\
& -\sum_{n=1}^{N} \Lambda^{T}\left(\iint_{S_{\mathrm{ie}}} \Upsilon_{\mathrm{en}} \Upsilon_{\mathrm{sen}}^{T} d S_{\mathrm{ie}}\right) \vec{p}_{\mathrm{en}}=F_{s} .
\end{aligned}
$$

Based on (10) and (13), the fluid-structure coupling model of control valve can be described as

$$
\begin{gathered}
{\left[\begin{array}{ll}
\rho \xi & \delta \\
M & 0
\end{array}\right]\left[\begin{array}{l}
\frac{\partial^{2} \Gamma}{\partial t^{2}} \\
\frac{\partial^{2} \vec{p}}{\partial t^{2}}
\end{array}\right]} \\
+\left[\begin{array}{ll}
0 & \varphi \\
C & 0
\end{array}\right]\left[\begin{array}{l}
\frac{\partial \Gamma}{\partial t} \\
\frac{\partial \vec{p}}{\partial t}
\end{array}\right] \\
+\left[\begin{array}{cc}
0 & \phi \\
K_{F} & -\xi^{T}
\end{array}\right]\left[\begin{array}{l}
\Gamma \\
\vec{p}
\end{array}\right]=\left[\begin{array}{l}
F_{i} \\
F_{s}
\end{array}\right],
\end{gathered}
$$

with

$$
\begin{gathered}
\delta=\frac{\rho}{K} \iiint_{\Omega} \Upsilon \Upsilon^{T} d \Omega, \\
\xi=\left(\iint_{S_{i}} \Upsilon_{s}^{T} d S_{i}\right) \Lambda, \\
\phi=\iiint_{\Omega} \nabla \Upsilon \cdot \nabla \Upsilon^{T} d \Omega, \\
\varphi=\frac{1}{\sqrt{K / \rho}} \iint_{S_{r}} \Upsilon \Upsilon^{T} d S_{r} .
\end{gathered}
$$

\section{Influence Analyses of the Fluid-Structure Coupling}

In this section, numerical simulations utilizing ANSYS, CFX, and Workbench were performed. In the analysis, a time step of $0.0005 \mathrm{~s}$ was used. A compressible, ideal gas flow was assumed for simulations. Inflow boundary conditions based on an inlet total pressure of $2 \mathrm{MPa}$ and a temperature of $240^{\circ} \mathrm{C}$ were specified at the inlet plane of the control valve. At the outflow plane of the control valve, a flow rate of $20 \mathrm{~kg} / \mathrm{s}$ was maintained. The reference pressure of the model environment was normal atmospheric pressure. The initial velocity vector was zero. And the mean residual of the convergence of the solution was less than 0.001 .

Figure 3 shows the general structure of the flow field through the control valve, which depicts the streamlines without fluid-structure coupling. For the time period of the simulation, about 32 cycles of data were collected. The progression of the simulation at intervals of $T / 4$ is shown in Figure 3. When taking the fluid-structure coupling into account, the general structure of the flow field is shown in Figure 4. As it is seen from Figures 3 and 4, after considering 


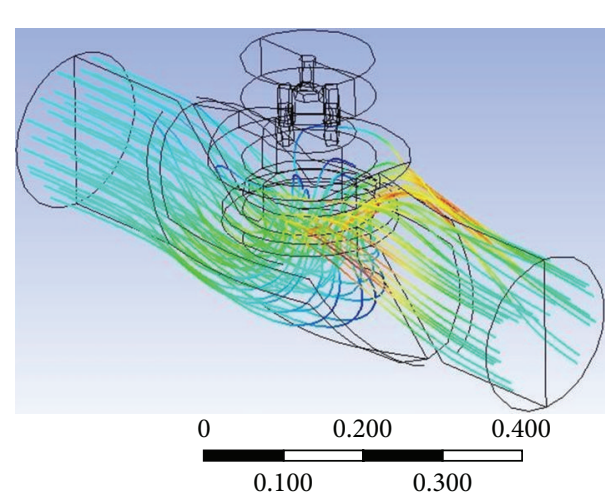

(m)

(a) $T / 4$

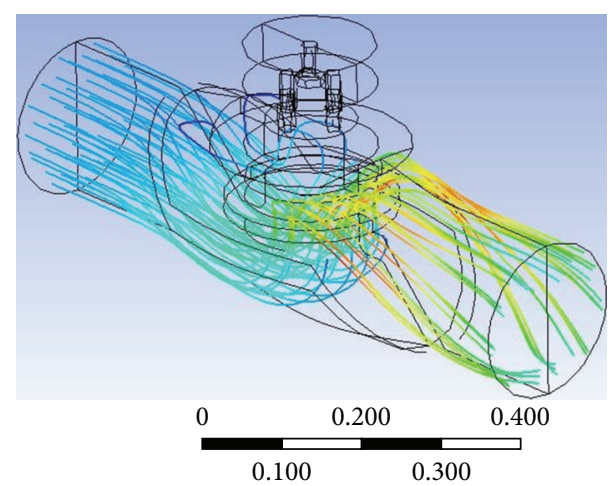

(m)

(c) $3 T / 4$
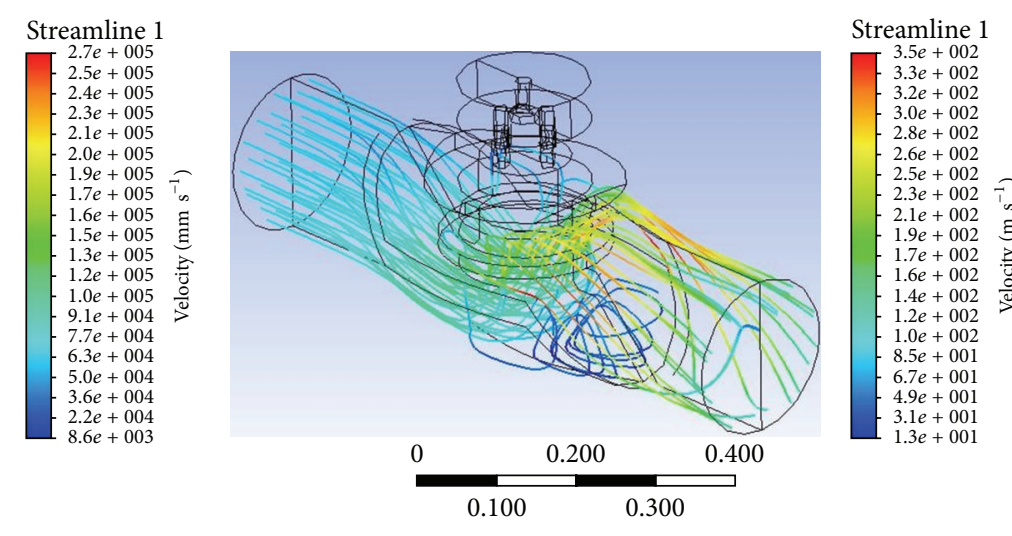

(m)

(b) $2 T / 4$
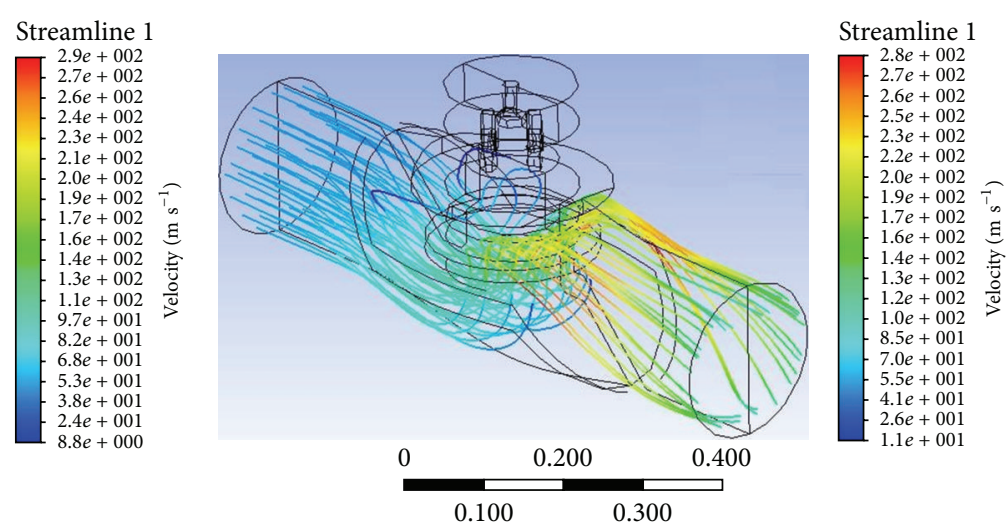

(m)

(d) $\mathrm{T}$

FIGURE 3: Streamlines without fluid-structure coupling.

the fluid-structure coupling, some of the secondary flow structures present in the corner regions of the valve housing, and the large recirculation develops in the bottom and upper portion of the valve body as the flow negotiates the transition from the valve assembly to the valve outlet. The flow sharply accelerates around the valve seat region.

The unsteady flow, as stated in Figure 4, causes pressure fluctuations with random and impulsive wave forms. The pressure distributions of the flow field through the control valve, considering and not considering the fluid-structure coupling, are shown in Figure 5. The peak pressure presented in Figure 5(a) is $1.9 \mathrm{MPa}$, while the peak pressure that appeared in Figure 5(b) is $2.3 \mathrm{MPa}$. Furthermore, the maximum pressure position shown in Figure 5(b) is different from that shown in Figure 5(a). In order to verify the influence of fluid-structure coupling on the gas pressure regulation of the control valve, a sine pressure with an amplitude of $1 \mathrm{MPa}$ and an initial value of $1 \mathrm{MPa}$ was specified at the inlet of the control valve. Figure 6 gives time-history plots of outlet pressure changes of control valve as compared to the inlet pressure changes without considering fluid-structure coupling. The outlet pressure can follow the inlet pressure signal well, which does not have obvious oscillations. When taking the fluid-structure coupling into account, the timehistory plots of outlet pressure changes of control valve as compared to the inlet pressure changes are shown in Figure 7. The simulation process with fluid-structure coupling has obvious pressure oscillations that are far greater than those obtained from the simulation process without fluid-structure coupling. As a result, the coupled oscillations of the flow in the control valve are maintained at certain operating conditions, and the fluid force acting on the valve plug becomes a random and pulse-like wave form, as shown in Figure 8. This fluid force is added to the driving force of the control valve, which brings about the result that the resultant force may be greater or less than the control force used to adjust the valve opening, and consequently, the control precision of the control valve is reduced.

Table 1 shows the natural frequencies obtained by the simulation at different valve opening positions. When taking the fluid-structure coupling into account, the low-order natural frequencies of the plug assembly of the control valve decrease. The first-order natural frequencies at $25 \%$ valve opening, $50 \%$ valve opening, and $75 \%$ valve opening are reduced by 


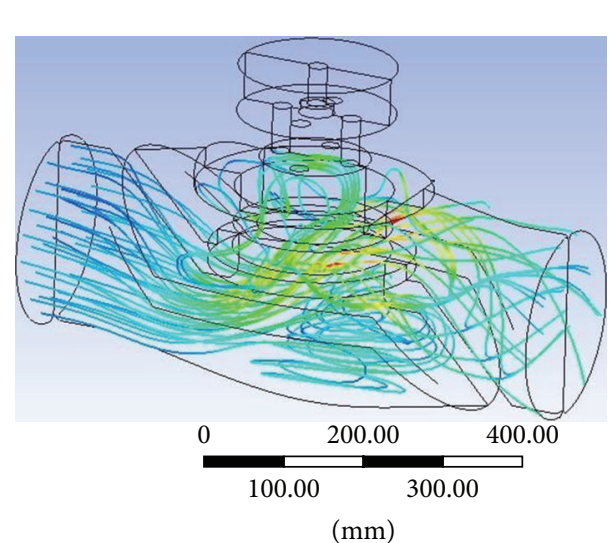

(a) $T / 4$

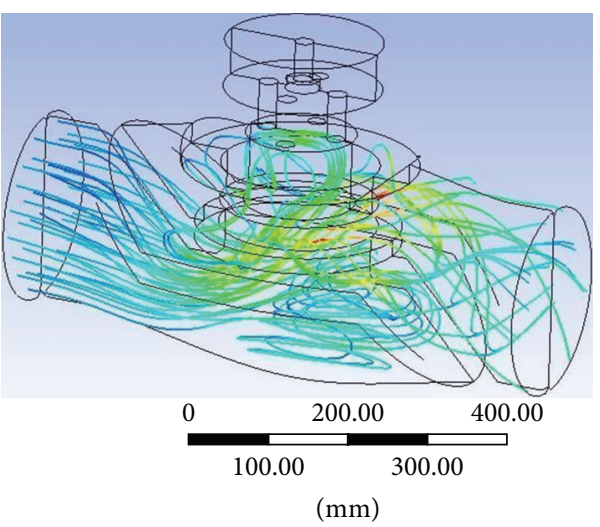

(c) $3 T / 4$
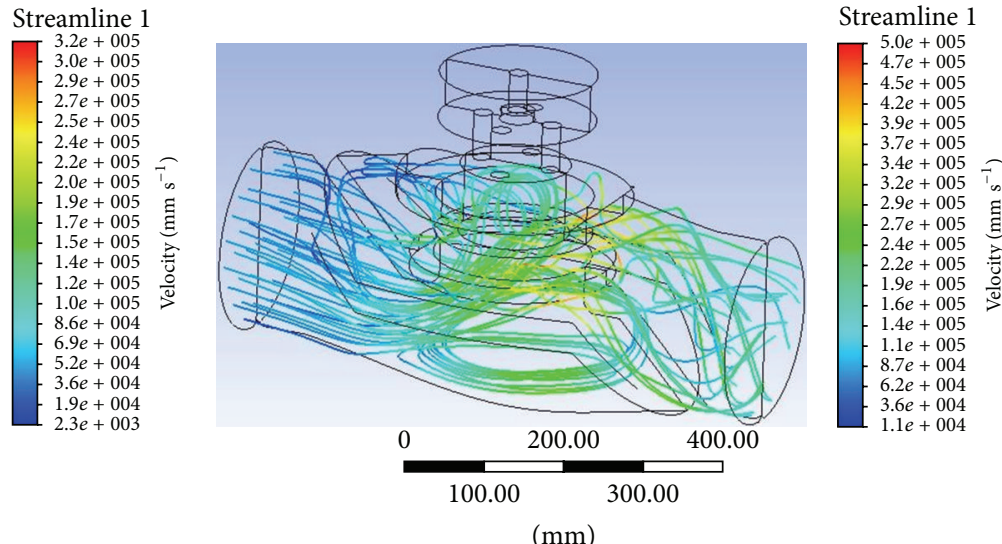

(b) $2 T / 4$
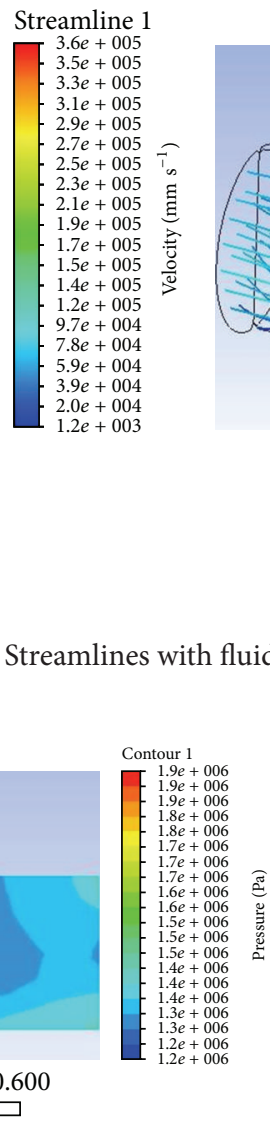

$\mathrm{m}$

(a) Not considering fluid-structure coupling

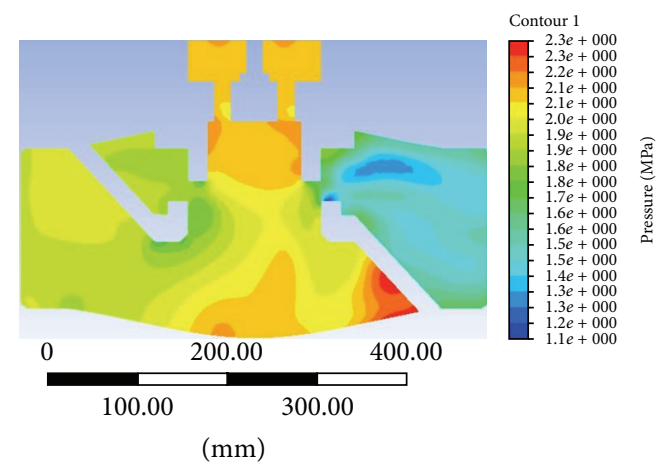

(b) Considering fluid-structure coupling

FIgURE 5: Pressure distributions.

$11.1 \%, 7.0 \%$, and $3.8 \%$, respectively. As a result, the vibrations become easy to excite due to the pressure fluctuations caused by the fluid-structure coupling. At the same time, the valve plug vibration affects the pressure fluctuation. The pressure fluctuation increases when the valve plug vibration increases, and in some cases with very small valve opening ratios, the valve plug hits the valve seat.

\section{Conclusions}

Fluid-structure interaction between the structure and the fluid system at the control valve has to be taken into account for the analysis of the control valve characteristics. This is extremely useful in a better understanding of the detailed flow physics that occur in control valves. The general 
TABLE 1: Natural frequencies of the plug assembly of the control valve.

\begin{tabular}{|c|c|c|c|c|}
\hline Valve opening & Order & $\begin{array}{c}\text { Natural frequency without } \\
\text { coupling } f(\mathrm{~Hz})\end{array}$ & $\begin{array}{l}\text { Natural frequency with } \\
\text { coupling } f_{s}(\mathrm{~Hz})\end{array}$ & $\begin{array}{c}\text { Ratio }\left(f_{s}-f\right) / f \\
(\%) \\
\end{array}$ \\
\hline \multirow{5}{*}{$25 \%$} & 1 & 89.822 & 79.901 & -11.1 \\
\hline & 2 & 1158.6 & 1158.4 & 0.0 \\
\hline & 3 & 3387.1 & 3387.3 & 0.0 \\
\hline & 4 & 3388.0 & 3388.3 & 0.0 \\
\hline & 5 & 6221.6 & 6221.3 & 0.0 \\
\hline \multirow{5}{*}{$50 \%$} & 1 & 107.84 & 100.32 & -7.0 \\
\hline & 2 & 1347.5 & 1347.8 & 0.0 \\
\hline & 3 & 3979.9 & 3980.1 & 0.0 \\
\hline & 4 & 3981.7 & 3982.5 & 0.0 \\
\hline & 5 & 8549.4 & 8552.3 & 0.0 \\
\hline \multirow{5}{*}{$75 \%$} & 1 & 145.56 & 140.10 & -3.8 \\
\hline & 2 & 1668.3 & 1669.0 & 0.0 \\
\hline & 3 & 4375.8 & 4376.0 & 0.0 \\
\hline & 4 & 4376.7 & 4376.5 & 0.0 \\
\hline & 5 & 9899.1 & 9899.3 & 0.0 \\
\hline
\end{tabular}

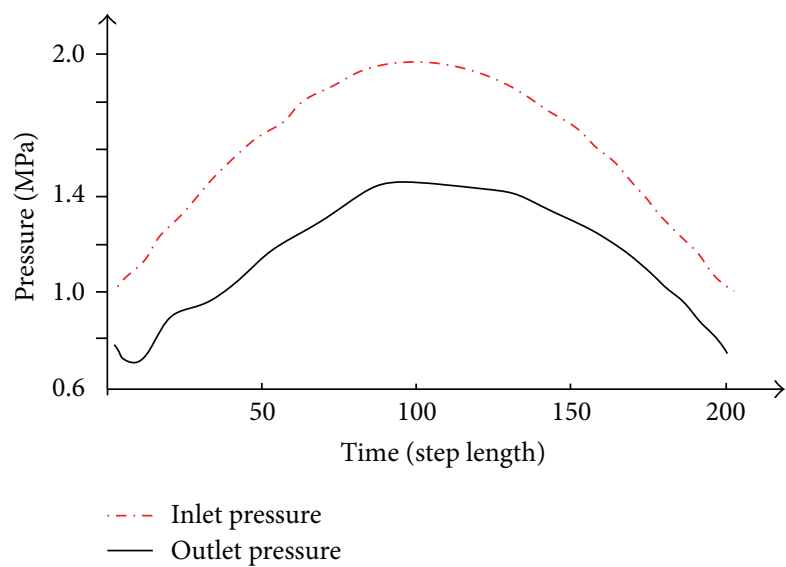

FIGURE 6: Pressure response plots (without considering fluid-structure coupling).

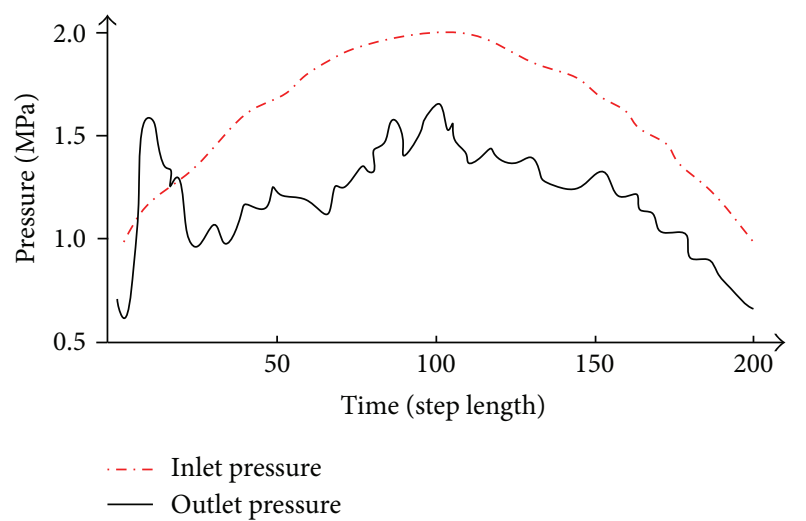

FIGURE 7: Pressure response plots (considering fluid-structure coupling).

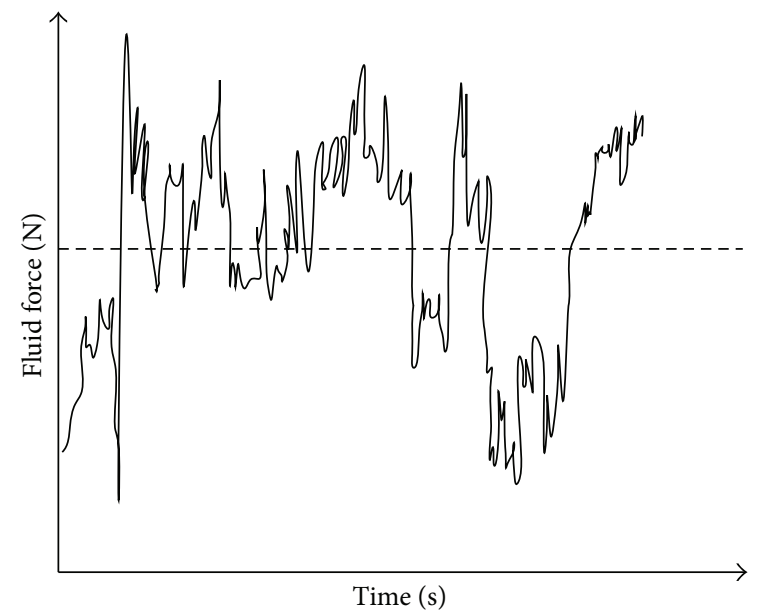

FIGURE 8: Time history of the fluid force on the valve plug.

structure of the flow field through the control valve, the valve plug vibration, and the pressure regulation performance are affected by the fluid-structure coupling. The unsteady fluid force due to the coupled oscillations of the flow in the control valve is added to the driving force of the control valve, which brings about the result that the resultant force may be greater or less than the control force used to adjust the valve opening, and consequently, the control precision of the control valve is reduced.

\section{Acknowledgments}

This work is supported by the National Natural Science Foundation of China (Grant no. 51305234), Special Funds for Postdoctoral Innovative Projects of Shandong Province (no. 
201103033), Independent Innovation Foundation of Shandong University (IIFSDU no. 2011GN045), and Key Laboratory of High-Efficiency and Clean Mechanical Manufacture at Shandong University of Education Ministry.

\section{References}

[1] Y.-D. Xie, Y.-J. Liu, and Y. Wang, "Design of cascade-smith hybrid control structure for pressure control," Key Engineering Materials, vol. 419-420, pp. 797-800, 2010.

[2] K. Yonezawa, Y. Toyohira, T. Nagashima et al., "An experimental study of unsteady transonic flow in a steam control valve with simple model," Transactions of the Japan Society of Mechanical Engineers B, vol. 74, no. 2, pp. 303-309, 2008.

[3] R. Morita, F. Inada, M. Mori, K. Tezuka, and Y. Tsujimoto, "CFD simulations and experiments of flow fluctuations around a steam control valve," Journal of Fluids Engineering, Transactions of the ASME, vol. 129, no. 1, pp. 48-54, 2007.

[4] K. Yonezawa, R. Ogawa, K. Ogi et al., "Flow-induced vibration of a steam control valve," Journal of Fluid and Structures, vol. 35, pp. 76-88, 2012.

[5] A. Misra, K. Behdinan, and W. L. Cleghorn, "Self-excited vibration of a control valve due to fluid-structure interaction," Journal of Fluids and Structures, vol. 16, no. 5, pp. 649-665, 2002.

[6] T. Araki, Y. Okamoto, and F. Otomo, "Fluid-induced vibration of steam control valves," Toshiba Review, vol. 36, no. 7, pp. 648656, 1981.

[7] K. Yonezawa, Y. Toyohira, T. Nagashima et al., "An experimental study of unsteady transonic flow in a steam control valve with simple model," Journal of Environment and Engineering, vol. 5, no. 1, pp. 134-143, 2010.

[8] N. S. Akbar and S. Nadeem, "Numerical and analytical simulation of the peristaltic flow of Jeffrey fluid with Reynold's model of viscosity," International Journal of Numerical Methods for Heat and Fluid Flow, vol. 22, no. 4, pp. 458-472, 2012.

[9] N. S. Akbar and S. Nadeem, "Mixed convective magnetohydrodynamic peristaltic flow of a Jeffrey nanofluid with newtonian heating," Zeitschrift Für Naturforschung A, vol. 68, pp. 433-441, 2013.

[10] N. S. Akbar and S. Nadeem, "Characteristics of heating scheme and mass transfer on the peristaltic flow for an Eyring-Powell fluid in an endoscope," International Journal of Heat and Mass Transfer, vol. 55, no. 1-3, pp. 375-383, 2012.

[11] N. S. Akbar and S. Nadeem, "Simulation of heat transfer on the peristaltic flow of a Jeffrey-six constant fluid in a diverging tube," International Communications in Heat and Mass Transfer, vol. 38, no. 2, pp. 154-159, 2011.

[12] N. S. Akbar and S. Nadeem, "An analytical and numerical study of peristaltic transport of a Johnson-Segalman fluid in an endoscope," Chinese Physics B, vol. 22, no. 1, pp. 1-9, 2013.

[13] J. Deng, X.-M. Shao, X. Fu, and Y. Zheng, "Evaluation of the viscous heating induced jam fault of valve spool by fluid-structure coupled simulations," Energy Conversion and Management, vol. 50, no. 4, pp. 947-954, 2009.

[14] D. Zhang, A. Engeda, J. R. Hardin, and R. H. Aungier, "Experimental study of steam turbine control valves," Proceedings of the Institution of Mechanical Engineers C, vol. 218, no. 5, pp. 493508, 2004.

[15] K.-J. Bathe, H. Zhang, and S. Ji, "Finite element analysis of fluid flows fully coupled with structural interactions," Computers and Structures, vol. 72, no. 1, pp. 1-16, 1999.
[16] H. Kohno and K.-J. Bathe, "A nine-node quadrilateral FCBI element for incompressible fluid flows," Communications in Numerical Methods in Engineering, vol. 22, no. 8, pp. 917-931, 2006.

[17] K.-J. Bathe and H. Zhang, "Finite element developments for general fluid flows with structural interactions," International Journal for Numerical Methods in Engineering, vol. 60, no. 1, pp. 213-232, 2004 


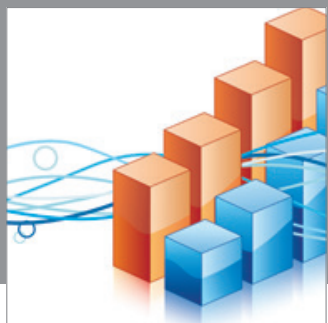

Advances in

Operations Research

mansans

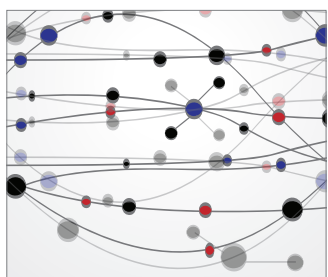

The Scientific World Journal
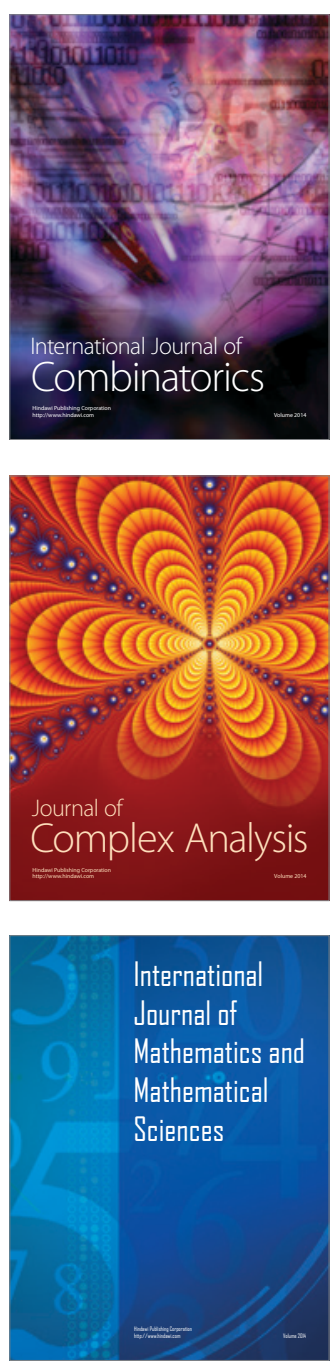
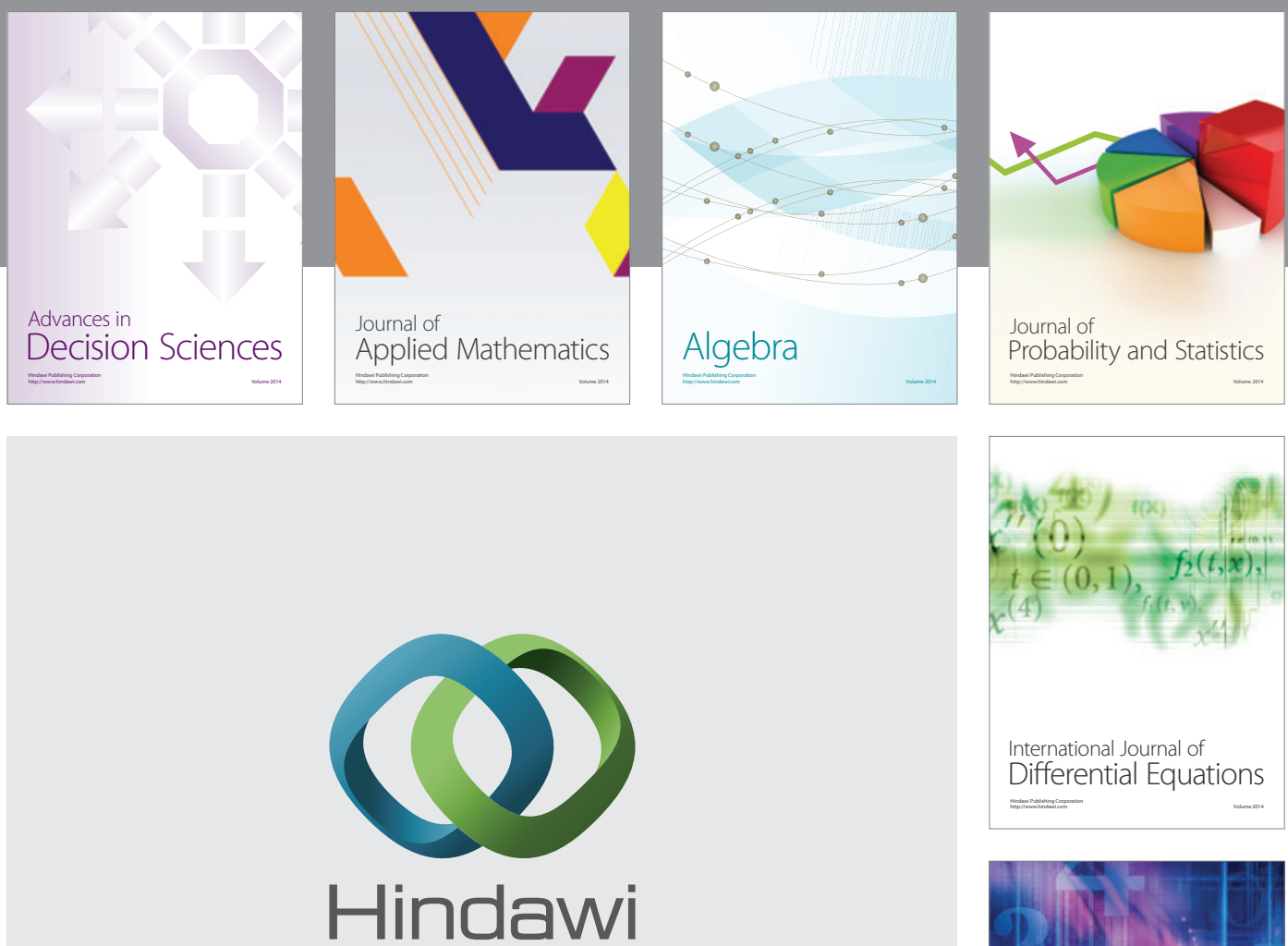

Submit your manuscripts at http://www.hindawi.com
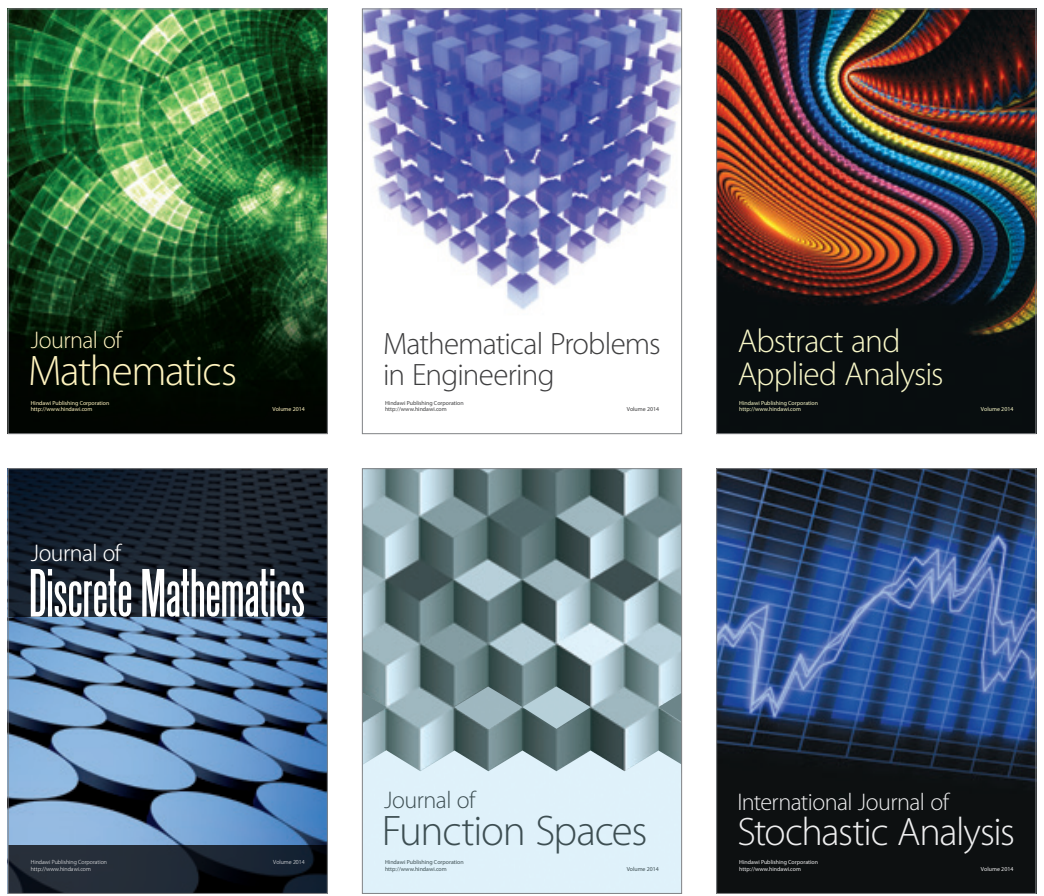

Journal of

Function Spaces

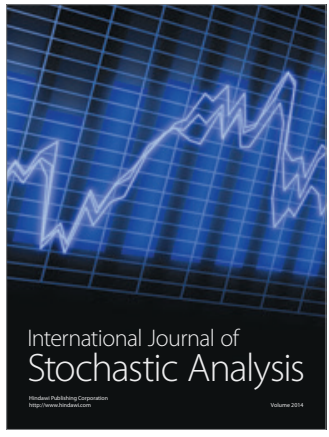

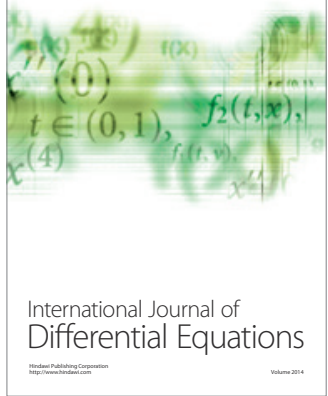
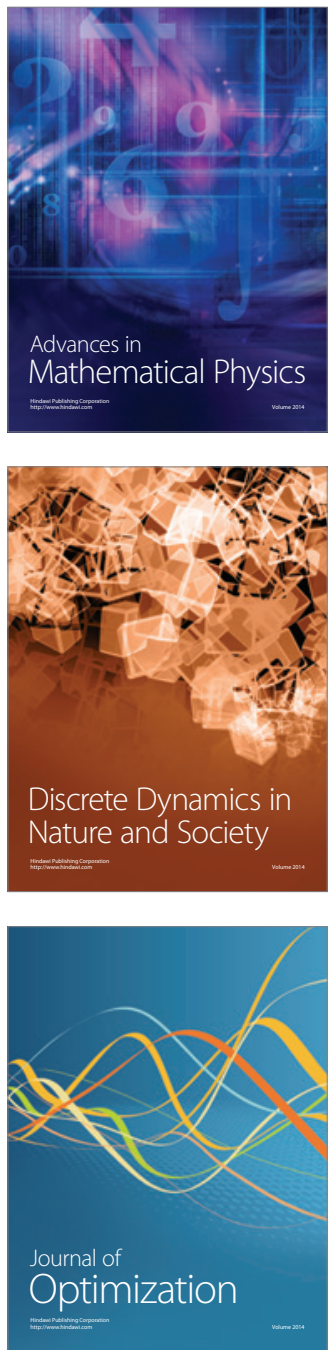